\title{
THE HURRICANE IMAGING RADIOMETER: PRESENT AND FUTURE
}

\author{
Timothy L. Miller ${ }^{1}$, M. W. James ${ }^{1}$, J. B. Roberts ${ }^{1}$, S. K. Biswas ${ }^{1}$, D. Cecil ${ }^{1}$, W. L. Jones ${ }^{2}$, J. Johnson ${ }^{2}, S$.

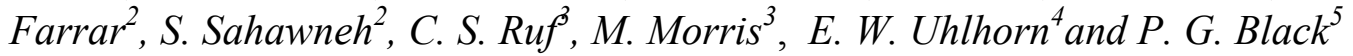 \\ ${ }^{1}$ NASA/MSFC, Earth Science Office, Huntsville, AL \\ ${ }^{2}$ EECS Dept., University of Central Florida, Orlando, FL \\ ${ }^{3}$ AOSS Dept., University of Michigan, Ann Arbor, MI \\ ${ }^{4}$ NOAA, Atlantic Oceanographic and Meteorological Laboratory (AOML) \\ ${ }^{5}$ SAIC Inc., Naval Research Laboratory, Monterey, CA
}

\begin{abstract}
The Hurricane Imaging Radiometer (HIRAD) is an airborne passive microwave radiometer designed to provide high resolution, wide swath imagery of surface wind speed in tropical cyclones from a low profile planar antenna with no mechanical scanning. Wind speed and rain rate images from HIRAD's first field campaign (GRIP, 2010) are presented here followed, by a discussion on the performance of the newly installed thermal control system during the 2012 HS3 campaign. The paper ends with a discussion on the next generation dual polarization HIRAD antenna (already designed) for a future system capable of measuring wind direction as well as wind speed.
\end{abstract}

Index Terms - HIRAD, hurricane wind speed, imaging radiometer.

\section{INTRODUCTION}

The Hurricane Imaging Radiometer (HIRAD) is an airborne passive microwave synthetic aperture radiometer designed to provide wide swath images of ocean surface wind speed under heavy precipitation and, in particular, in tropical cyclones. The HIRAD instrument operates at 4, 5, 6 and 6.6 $\mathrm{GHz}$ and uses interferometric signal processing to synthesize a pushbroom imager from a low profile planar antenna with no mechanical scanning. HIRAD flew on high-altitude aircraft over hurricanes Earl and Karl during NASA's GRIP (Genesis and Rapid Intensification Processes) campaign in August - September of 2010 and recently during NASA's HS3 (Hurricane and Severe Storm Sentinel) campaign in November 2012. From these flights, sample results are presented that demonstrate that HIRAD can provide fourfrequency images of upwelling brightness temperature $(\mathrm{Tb})$ over a $\sim 60 \mathrm{~km}$ swath width with $\sim 3 \mathrm{~km}$ spatial resolution using an image reconstruction algorithm [1]. From these Tb data, ocean surface wind speed and column averaged atmospheric liquid water content (or rain rate) have been retrieved across the swath and examples are presented for hurricane Earl.

To improve the radiometric calibration stability of the HIRAD instrument, various hardware design changes have been implemented during the HS3 mission. These include a thermal control system and an improved (more stable) set of local oscillators. In-flight temperature measurements are presented here that demonstrate improved instrument thermal stability has been achieved.

Finally, the present HIRAD instrument is capable of measuring only horizontal polarization of the ocean brightness emissions. A new dual-pol phased array antenna prototype has been built and tested, which will improve the $\mathrm{Tb}$ measurement accuracy over a wide swath and enable the measurement of ocean surface wind direction as well as wind speed.

\section{HIRAD INSTRUMENT OVERVIEW}

The current HIRAD planar antenna is composed of linear arrays of stacked multi-resonant radiators, operating at 4,5 , 6, and $6.6 \mathrm{GHz}$ [2]. Each linear array, viewed as an individual fan beam antenna element, is placed in an optimum thinned array configuration to produce the interferometer baselines needed for aperture synthesis. Individual receivers after each fan beam element contain internal hot and cold calibration loads. A single correlated noise diode is also distributed to all receivers to calibrate the interferometric correlation measurements [3]. HIRAD's major components consist of the integrated stacked patch antenna array, analog receivers with integrated calibration sources, real time digital signal processing for frequency sub-banding, RFI mitigation and interferometric crosscorrelation, command and data handling (C\&DH), Local Oscillator (LO) and noise diode (for calibration) distribution, and the Power Distribution Unit (PDU), which includes thermal control for the instrument. The current 


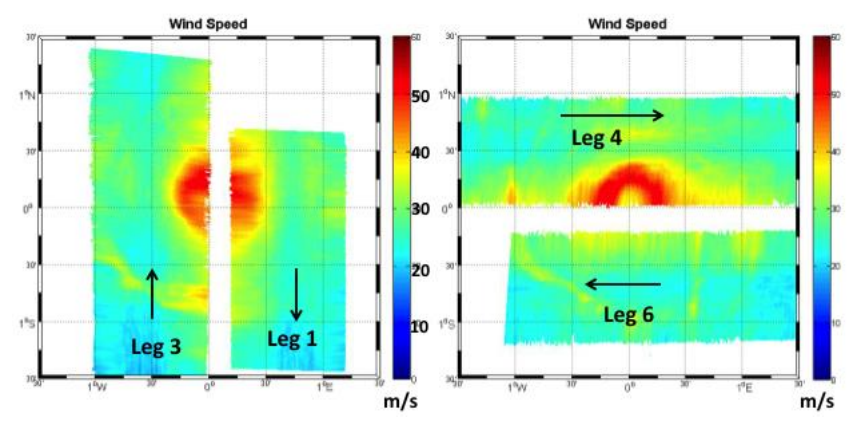

Figure 1: HIRAD wind speed $(\mathrm{m} / \mathrm{s})$ images retrieved from 5 $\mathrm{GHz} \mathrm{Tb}$ observations during hurricane Earl overflight on 12 Sept, 2010.
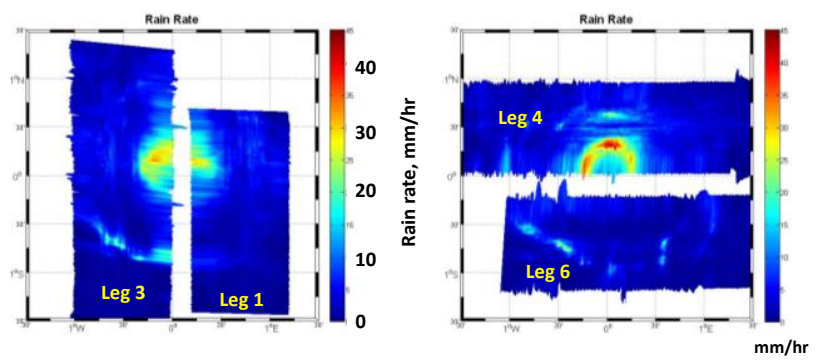

Figure 2: HIRAD rain rate $(\mathrm{mm} / \mathrm{hr})$ images retrieved from 5 $\mathrm{GHz} \mathrm{Tb}$ observations during hurricane Earl overflight on 12 Sept, 2010.

instrument is capable of measuring only the horizontal polarization of the incident radiation.

\section{GRIP CAMPAIGN (2010)}

Following an accelerated development schedule, HIRAD was flown for the first time on a NASA WB-57 aircraft over hurricanes Earl and Karl during the 2010 NASA GRIP campaign. Due to the temperature dependent drift in the HIRAD data and absence of thermal control system during GRIP the calibration of the instrument's Tb images posed many challenges. So far, only the $5 \mathrm{GHz} \mathrm{Tb}$ are produced with acceptable quality from the GRIP data. Figure 1 shows the single frequency $(5 \mathrm{GHz}$ only) wind speed $(\mathrm{m} / \mathrm{s})$ retrieval images in storm-centric coordinates from the Hurricane Earl overflight during 1-2 September 2010. South (leg 1) and north (leg 3) bound legs are shown on the left panel and east (leg 4) \& west (leg 6) bound legs are shown on the right panel of the figure, respectively. Figure 2 shows the corresponding images of columnar average rain rate in $\mathrm{mm} / \mathrm{hr}$.

In Figure 3 the HIRAD wind speed retrievals for leg 4 are compared with nearly simultaneous observations by the Stepped Frequency Microwave Radiometer (SFMR) flying on a NOAA P3 aircraft. In the retrieved HIRAD wind speed image (top panel) the SFMR flight track is marked with a black line. SFMR only measures directly beneath the aircraft, not along a swath. The bottom panel compares the

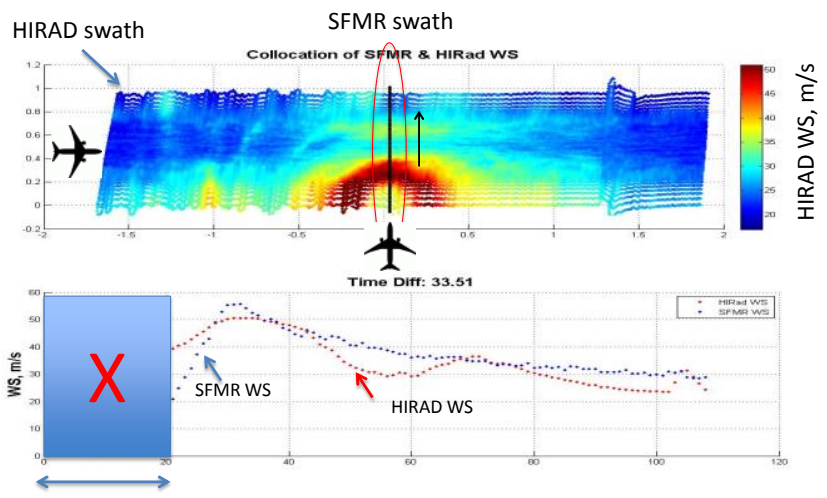

Figure 3: Comparison of HIRAD retrieved wind speed with the SFMR observed wind speed during hurricane Earl overflight.

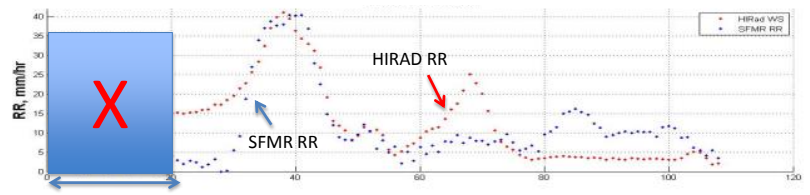

Figure 4: Comparison of HIRAD retrieved rain rate with the SFMR observed rain rate during hurricane Earl overflight.

wind speed retrievals from SFMR (blue dots) with the corresponding retrievals from HIRAD (red dots). The horizontal axis of the bottom panel is the pixel number from south to north. The eyewall rain column contaminates retrievals inside the eye near the swath edge, leading to overestimates of surface wind speed there by HIRAD. There is approximately 34 minutes time difference between the two observations, which introduces the possibility of differences due to system motion and evolution. Figure 4 shows the rain rate $(\mathrm{mm} / \mathrm{hr})$ retrievals for the same pixels. This dataset is available online at [4]. The comparisons outside of the eye are generally encouraging.

\section{THERMAL CONTROL SYSTEM PERFORMANCE}

From our previous experience during GRIP it was found that the HIRAD instrument exhibits strong physical temperature dependent calibration drift. Fig 5(a) shows a time-series plot for all 10 receiver physical temperatures during a flight over hurricane Karl in 2010 GRIP campaign. Due to the absence of a thermal control system, the temperatures are found to drift throughout the flight. Prior to the HS3 campaign in 2012, a thermal control system was added [see Fig. 5(b)] which stabilized the temperature drift. Note that in Fig. 5(b) a similar drift as that of Fig 5(a) in temperature was observed during 22:02 - 23:44 UTC 5 November 2012, because the thermal control was not activated during that time. The thermal control system was activated around 23:44 UTC, and the system was able to stabilize the receiver temperatures during 08:13 - 16:43 UTC 6 November 2012. 


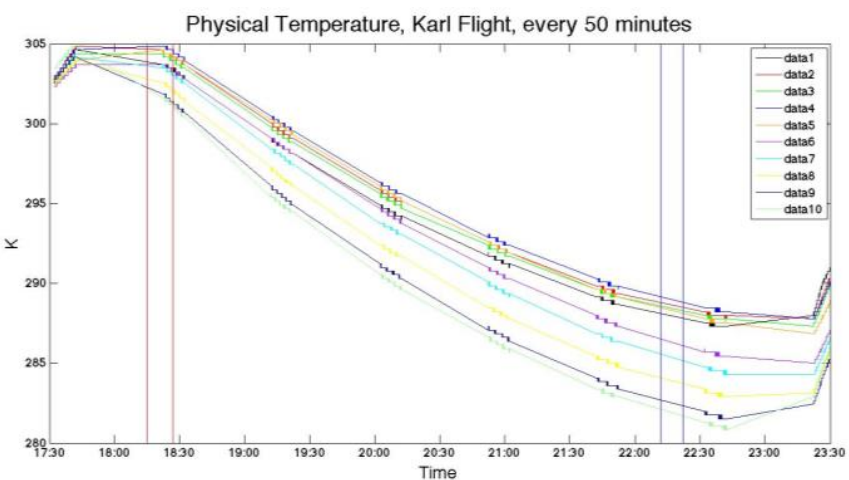

(a) Receiver temperatures during a typical flight (GRIP

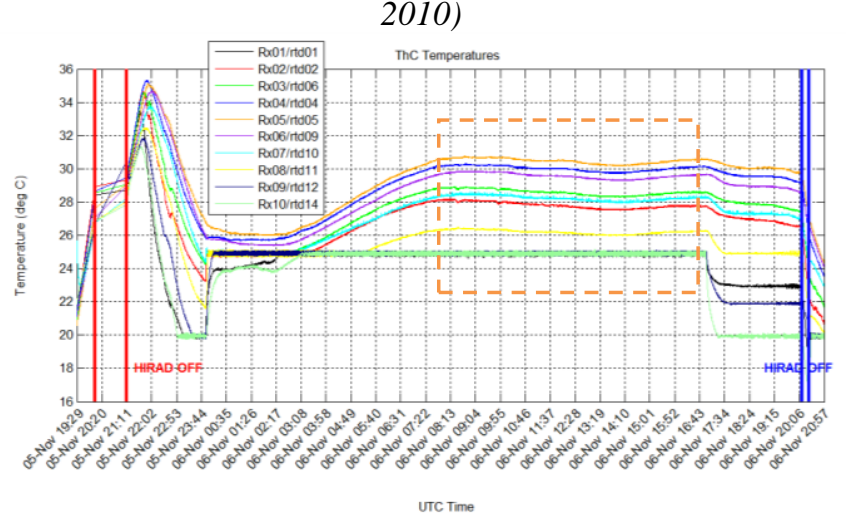

(b) Improved temperature stabilization during recent HS3 hurricane campaign.

Figure 5: Physical temperature time-series of all 10 HIRAD receivers during high-altitude flights. A new thermal control system, operational during HS3 campaign, resulted in stabilized receiver temperatures (orange box).

One science flight was made during the 2012 HS3 campaign with this new thermal system in place. The results from this flight demonstrate the proper working of the thermal control sub-system. We continue to see small thermal control system artifacts. To mitigate this, we plan to make additional refinements to the thermal control system and make additional laboratory tests before the 2013 HS3 deployment.

\section{NEXT GENERATION HIRAD}

A new dual-polarized HIRAD system is being designed to measure wind direction in addition to speed. A dualpolarized phased array antenna prototype, developed via a SBIR (Small Business Innovation Research), has been built and tested [see Fig. 6]. The new system will also improve the $\mathrm{Tb}$ measurement accuracy over a wide swath and similar technology may eventually be used on a satellite platform to extend the dynamical range of Ocean Surface Wind Vector (OSV) observation from space.

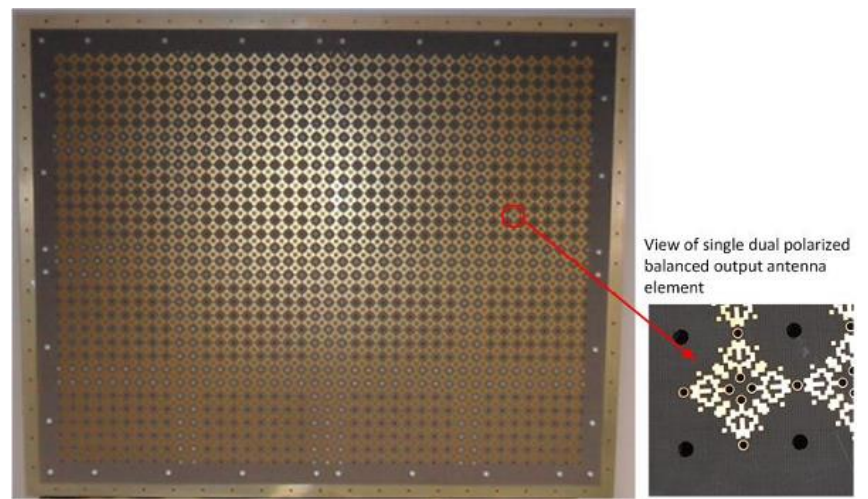

Figure 6: New dual-pol antenna prototype for next generation HIRAD system.

\section{SUMMARY}

This paper describes the Hurricane Imaging Radiometer (HIRAD), a new airborne passive microwave radiometer designed to provide high resolution wide swath image of surface wind speed in tropical cyclones from a low profile planar antenna with no mechanical scanning. Wind speed and rain rate images from HIRAD's first field campaign (GRIP, 2010) are presented here followed by a discussion on the performance of the newly installed thermal control system during 2012 HS3 campaign. Finally the paper ends with a discussion on the already designed next generation dual polarization HIRAD antenna for a future system capable of measuring wind direction as well as wind speed.

\section{REFERENCES}

[1] Ruf, Christopher; Roberts, J. Brent; Biswas, Sayak; James, Mark W. and Miller, Timothy, "Calibration and Image Reconstruction for the Hurricane Imaging Radiometer (HIRAD)" Proc. IGARSS 2012, Munich, 22-27 July 2012.

[2] Bailey, M.C., R. Amarin, J. Johnson, P. Nelson, M. James, D. Simmons, C. Ruf, L. Jones and X. Gong, "MultiFrequency Synthetic Thinned Array Antenna for the Hurricane Imaging Radiometer," Trans. Antennas Propagat., 58(8), doi:10.1109/TAP.2010.2050453, 2010.

[3] Ruf, C., R. Amarin, M.C. Bailey, B. Lim, R. Hood, M. James, J. Johnson, L. Jones, V. Rohwedder and K. Stephens, "The hurricane imaging radiometer - An octave bandwidth synthetic thinned array radiometer," Proc. IGARSS 2007, Barcelona, 23-27 July 2007.

[4] http://ghrc.nsstc.nasa.gov/hydro/details.pl?ds=griphirad 